\title{
Corrections to the rate equation approximation for dynamic considerations in a semiconductor laser
}

\author{
Kerry Vahala ${ }^{\text {a) }}$ \\ California Institute of Technology, 128-95, Pasadena, California 91125
}

(Received 11 February 1986; accepted for publication 26 March 1986)

Corrections to the rate equation approximation are derived and applied to a semiconductor laser. Whereas these corrections do not affect the operating point of the device, they do alter the dynamic operation. To first order the correction produces a renormalization of familiar dynamic parameters. This renormalization, in turn, leads to a $20 \%$ correction to the field spectrum linewidth formula.

Semiconductor lasers (SL's) fabricated in the GaAs (AlGaAs) or InGaAsP (InP) systems serve as sources and local oscillators in optical communication systems based on silica fiber. Their dynamic and fluctuation properties are, therefore, of considerable importance. To date, these properties have been studied theoretically using field and carrier equations of motion based on the rate equation approximation. This well-known approximation adiabatically eliminates the polarization as a dynamic variable in the laser equations of motion. This letter shows that, in fact, this approximation does not hold in a semiconductor laser for consideration of dynamics and noise. Corrections to the dynamic equations caused by the breakdown of the rate equation approximation will be derived. To first order these corrections result in a renormalization of familiar dynamic quantities in terms of $g_{\Omega}$ and $\mu_{s}$, the derivative of gain and refractive index with respect to frequency. We show how certain well-known expressions for linewidth and direct-modulation response are modified by these corrections. A detailed discussion of these results will appear elsewhere. ${ }^{\text {I }}$

We begin by considering the rate equation approximation as normally applied to adiabatically eliminate the polarization in the laser equations of motion. For pedagogical reasons we consider a two-band model in which $k$ selection holds. We further assume that polarization decay can be modeled in terms of a single time constant. For such a model the polarization amplitude equation of motion, when integrated, has the following form ${ }^{2}$ :

$$
\begin{aligned}
P_{k}= & \int_{-\infty}^{t} i M_{k}^{*}\left[n_{k c}(z)-n_{k v}(z)\right] E(z) \\
& \quad x \exp \left\{\left[-i\left(\omega_{k}-\Omega_{L}\right)-\lambda_{k}\right](t-z)\right\} d z,
\end{aligned}
$$

where $P_{h}$ is the slowly varying polarization amplitude for an electron-hole pair having crystal wave vector $k, M_{k}^{*}$ is the dipole matrix element governing the transition, $n_{k c}\left(n_{k v}\right)$ is the occupancy of state $k$ in the conduction (valence) band (given by the quasi-Fermi distribution function), $E$ is the slowly varying complex lasing field amplitude (lasing frequency $\Omega_{L}$ ), $\hbar \omega_{k}$ is the energy separation of the electronhole pair, and $\lambda_{k}$ is the polarization dephasing rate. The rate equation approximation removes the dynamic variables $n_{k c}$, $n_{k}$, and $E$ to outside the integral in (1), leaving the following highly simplified expression for $P_{k}$ :

\footnotetext{
" This work was conducted while the author was a guest scientist at SEL in Stuttgart, West Germany
}

$$
P_{k}=\frac{i M_{k}^{*}\left(n_{k c}-n_{k v}\right) E}{i\left(\omega_{k}-\Omega_{L}\right)+\lambda_{k}},
$$

which is used to eliminate $P_{k}$ wherever it appears in the other laser equations of motion. The justification for this procedure is normally that $\lambda_{k}$ is sufficiently large to cause the exponential in (1) to act as a temporal sampling function. Using Eq. (2), optical gain $g$ and refractive index $\mu$ can be defined as follows ${ }^{2}$ :

$$
\begin{aligned}
& P_{\text {TOT }}^{(0)}=\sum_{k}-i M_{k} P_{k} \\
& \quad=\frac{1}{2}\left[g\left(N, \Omega_{L}\right)+i \frac{2 \Omega_{L}}{\mu_{0}} \mu\left(N, \Omega_{L}\right)\right] E, \\
& \frac{1}{2} g\left(N, \Omega_{L}\right)=\sum_{k} \frac{\lambda_{k}\left|M_{k}\right|^{2}\left(n_{k c}-n_{k v}\right)}{\left(\omega_{k}-\Omega_{L}\right)^{2}+\lambda_{k}^{2}}, \\
& \frac{\Omega_{L}}{\mu_{0}} \mu\left(N, \Omega_{L}\right)=-\sum_{\bar{k}} \frac{\left(\omega_{k}-\Omega_{L}\right)\left|M_{k}\right|^{2}\left(n_{k c}-n_{k v}\right)}{\left(\omega_{k}-\Omega_{L}\right)^{2}+\lambda_{k}^{2}},
\end{aligned}
$$

where $P_{\text {fOT }}^{(0)}$ is the total resonant contribution to polarization resulting from the rate equation approximation and $\mu_{0}$ is the nonresonant refractive index. The inversion or excitation parameter in these expressions has been taken as the carrier density $N$ rather than an equivalent representation in terms of either of the quasi-Fermi levels.

We now consider corrections to the rate equation approximation in the form of an asymptotic series. Consider integrating by parts repeatedly in Eq. (1). The first two such integrations yield

$$
\begin{aligned}
P_{k}= & \frac{i M_{k}^{*}\left(n_{k c}-n_{k v}\right) E}{D_{k}}-\frac{i M_{k}^{*}\left[\left(n_{k c}-n_{k v}\right) E\right]^{\prime}}{D_{k}^{2}} \\
& +\frac{1}{D_{k}^{2}} \int_{-\infty}^{I} i M_{k}^{*}\left[\left(n_{k c}(z)-n_{k v}(z)\right) E(z)\right]^{\prime \prime} \\
& \times e^{-D_{k}(1-z)} d z
\end{aligned}
$$

where $D_{k}=i\left(\omega_{k}-\Omega_{L}\right)+\lambda_{k}$ and [ ]' indicates differentiation with respect to the temporal variable. The first term in this series can be identified as the "rate equation" term [see Eq. (2) ]. The second term is a first order correction which gives the following first order correction to total resonant polarization, 


$$
\begin{aligned}
P_{\mathrm{TOT}}^{(1)} & =-\sum_{k} \frac{\left|M_{k}\right|^{2}\left[\left(n_{k c}-n_{k v}\right) E\right]^{\prime}}{D_{k}^{2}} \\
& =i \partial_{\Omega_{L}} \partial_{t} \sum_{k} \frac{\left|M_{k}\right|^{2}\left[\left(n_{k c}-n_{k k^{\prime}}\right) E\right]}{D_{k}} \\
& =i E \partial_{\Omega_{t}} \partial_{t}\left(\frac{g}{2}+i \frac{\Omega_{L}}{\mu_{0}} \mu\right)+i \bar{E} \partial_{\Omega_{L}}\left(\frac{g}{2}+i \frac{\Omega_{L}}{\mu_{0}} \mu\right),
\end{aligned}
$$

where the final equality results from Eqs. (4) and (5). If the expansion in Eq. (6) is continued, then successively higher order corrections to $P_{\text {тот }}$ can be calculated and then expressed in terms of $g\left(N, \Omega_{L}\right)$ and $\mu\left(N, \Omega_{L}\right)$ as was done in Eq. (7). The $n$th order correction is given by

$$
\begin{aligned}
P_{\mathrm{TOT}}^{(n)}= & \sum_{j=0}^{n}(i)^{n}\left(\begin{array}{c}
n \\
j
\end{array}\right) E^{(j)} \partial_{\Omega_{L}}^{n} \partial_{t}^{n-j} \\
& \times\left(\frac{g\left(N, \Omega_{L}\right)}{2}+i \frac{\Omega_{L}}{\mu_{0}} \mu\left(N, \Omega_{L}\right)\right) .
\end{aligned}
$$

It is clear from the form of Eq. (8) that none of these corrections will affect the dc operation of the laser. For consideration of device dynamics it is possible to show that only the first order correction $P_{\text {TOT }}^{(1)}$ is significant. ${ }^{\prime}$ We now investigate the effect of this correction on device dynamics and noise. Before proceeding, however, we note that although the above derivation involved a two-band model and a single time constant $\lambda_{k}^{-1}$ governing polarization decay, this expression is, in fact, rather general.'

Consider the first order correction $P_{\text {TOT }}^{(1)}$ and its affect on the field amplitude equation of motion, ${ }^{3}$

$$
\check{E}=(-i \Delta \Omega-\gamma) E+\Gamma P_{\mathrm{TOT}}^{(0)}+\Gamma P_{\mathrm{TOT}}^{(1)}+\mathscr{L},
$$

where $\Delta \Omega \equiv \Omega-\Omega_{L}$ ( $\Omega$ is the unpumped-cavity resonant frequency for the particular longitudinal mode of interest ), $\gamma$ is the cavity damping rate, $\Gamma$ is a confinement factor to account for incomplete spatial overlap of the field and polarization, and $\mathscr{L}$ is a Langevin noise source representing spontaneous noise into the mode. A partial linearization of this equation (i.e., linearize gain and refractive index and then extract steady state) yields

$$
\bar{E}=\frac{\Gamma}{2}\left(g_{N}+i \frac{2 \Omega_{L}}{\mu_{0}} \mu_{N}\right) N_{1} E+\Gamma P \text { Tor }+\mathscr{L},
$$

where $N=N_{0}+N_{1}, g(N) \doteq g\left(N_{0}\right)+g_{N} N_{1}$, and $\mu(N)$ $\doteq \mu\left(N_{0}\right)+\mu_{N} N_{1} . P_{\text {TOT }}^{\text {(1) }}$ can be approximated to a high degree of accuracy by

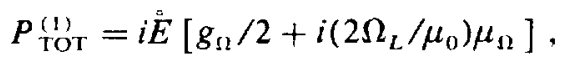

where for example, $g_{\Omega}=\partial_{\Omega_{L}} g\left(N_{0}, \Omega_{L}\right)$. (Note: The neglected terms here involve derivatives like $g_{N \Omega}$ and $\mu_{N \Omega}$ which can be shown to be negligible. ${ }^{4}$ ) If we substitute Eq. (11) into Eq. (10) it leads to a renormalization in this equation as follows:

$$
\begin{aligned}
\hat{E}= & \frac{\Gamma}{2} \frac{g_{N}+i\left(2 \Omega_{L} / \mu_{0}\right) \mu_{N}}{1-i(\Gamma / 2)\left[g_{\Omega}+i\left(2 \Omega_{L} / \mu_{0}\right) \mu_{n 1}\right]} N_{1} E \\
& +\frac{\mathscr{P}}{1-i(\Gamma / 2)\left[g_{\Omega}+i\left(2 \Omega_{L} / \mu_{0}\right) \mu_{\Omega}\right]}
\end{aligned}
$$

That is, the form of the equation is identical to its conventional rate equation approximation form but the magnitudes of differential quantities and the strength of the Langevin Force are renormalized. The small-signal carrier rate equation is affected similarly.

It is straightforward to show that the single-mode fieldspectrum linewidth $\Delta \omega$ and the direct modulation corner frequency $\omega_{R}$ (Ref. 5) are now given by

$$
\begin{aligned}
& \Delta \omega=\left[\Delta \omega_{\mathrm{ST}}\left(1+\alpha^{2}\right)\right] /(1+q-\alpha r)^{2}, \\
& \omega_{R}^{2}=\frac{g_{N} P}{\tau} \frac{1+q-\alpha r}{(1+q)^{2}+r^{2}},
\end{aligned}
$$

where $\Delta \omega_{\mathrm{ST}}$ is the Schawlow-Townes linewidth, $\alpha$ is the linewidth enhancement factor, ${ }^{\sigma-8} P$ is the lasing mode photon density, and $\tau+1 / 2 \gamma$ is the photon lifetime. Here we have also defined $q$ as $\Gamma \Omega_{l .} \mu_{\Omega} / \mu_{0}$ and $r$ as $\Gamma g_{s \Omega} / 2$. By setting $q$ and $r$ to zero in these expressions we get their conventional forms. For laser operation at the gain peak we have $r=0$ and these expressions have the following forms:

$$
\begin{aligned}
& \Delta \omega=\left[\Delta \omega_{\mathrm{ST}}\left(1+\alpha^{2}\right)\right] /(1+q)^{2}, \\
& \omega_{R}^{2}=\left(g_{N} P / \tau\right)[1 /(1+q)] .
\end{aligned}
$$

$q$ is related to the group and normal indices of refraction by $q=\Gamma\left(\mu_{g}-\mu_{0}\right) / \mu_{0}$, which follows from its definition. Taking $\mu_{g}=4.3, \mu_{0}=3.5$ (GaAs), and $\Gamma=0.5$ we estimate $q$ to be $0.11 .{ }^{9}$ This produces a negligible correction to $\omega_{R}$, but approximately a $20 \%$ reduction of $\Delta \omega$.

In conclusion, we have derived corrections to the rate equation approximation for a single-mode semiconductor laser. Although these corrections do not affect the operating point of the device, they do alter dynamics and noise. The first order correction leads to a renormalization of dynamic quantities as well as the Langevin source strength. For gain peak operation this produces a $20 \%$ shift in what was before the accepted form for linewidth; the modulation corner frequency has a negligible correction. We will consider the effect of this correction on other dynamic and spectral properties as well as higher order corrections in another publication.

The author is grateful for several stimulating discussions with Charles Kao and for financial support from SEL. The author also wishes to thank Leslie Westbrook for furnishing a preprint concerning measurement of $g_{v}$ and $\mu_{n}$.

\footnotetext{
'K. Vahala (unpublished).

${ }^{2}$ K. Vahala and A. Yariv, Phys. Rev. A 32, 345 (1985).

${ }^{3}$ M. Sargent, III, M. O. Scully, and W. E. Lamb, Jr., Laser Physics (Addison-Wesley, Reading, MA, 1974).

${ }^{4} \mathrm{~L}$. Westbrook (unpublished).

${ }^{5}$ K. Lau and A. Yariv, IEEE J. Quantum Electron. QE-21, 121 (1985).

${ }^{\circ} \mathrm{A}$. Mooradian, Phys. Today 38(5), 42 (1985).

${ }^{7}$ C. H. Henry, IEEE J. Quantum Electron. QE-18, 259 (1982).

${ }^{8}$ K. Vahala and A. Yariv, J. Quantum Electron QE-19, 1096, 1102 ( 1983 ).

${ }^{\circ}$ C. H. Henry, R. A. Logan, and K. A. Bertness, J. Appl. Phys. 52, 4453 (1981).
} 